\title{
Design of Fixed and Adaptive Companding Quantizer with Variable-Length Codeword for Memoryless Gaussian Source
}

\author{
Zoran H. PERIĆ ${ }^{1}$, Jelena R. NIKOLIĆ ${ }^{1}$, Aleksandar V. MOSIĆ ${ }^{1}$, \\ Marko D. PETKOVIĆ ${ }^{2}$ \\ ${ }^{1}$ Faculty of Electronic Engineering, University of Niš \\ Aleksandra Medvedeva 14, 18000 Niš, Serbia \\ ${ }^{2}$ Faculty of Sciences and Mathematics, University of Niš

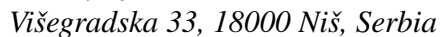 \\ e-mail: zoran.peric@elfak.ni.ac.rs, jelena.nikolic@elfak.ni.ac.rs, \\ mosicaca@yahoo.com,dexterofnis@gmail.com
}

Received: July 2011; accepted: April 2012

\begin{abstract}
The problem we address in this paper is the design of a quantizer that in comparison to the classical fixed-rate scalar quantizers provides more sophisticated bit rate reduction while restricting the class of quantizers to be scalar. We propose a switched variable-length code (VLC) optimal companding quantizer composed of two optimal companding scalar quantizers, the inner and the outer one, both designed for the memoryless Gaussian source of unit variance. Quantizers composing the proposed quantizer have a different codebook sizes and a different compressor functions. Particularly, we assume a smaller size of the inner quantizer's codebook in order to provide assignment of the shorter codewords to the high probability low amplitude speech samples belonging to the support region of the inner quantizer. We study the influence of codebook size of the inner and the outer quantizer on the Signal to Quantization Noise Ratio $(S Q N R)$. In such a manner the conclusion of the proposed quantizer significance in speech compression is distinctly shown in the paper. For the proposed quantizer model and its forward adaptive version the $S Q N R$ robustness analysis in a wide variance range is also presented in the paper. It is shown that our multi-resolution quantizer can satisfy G.712 Recommendation for high-quality quantization at the bit rate of $6.3 \mathrm{bit} / \mathrm{sample}$ achieving the compression of $1.7 \mathrm{bit} / \mathrm{sample}$ over the $\mathrm{G} .711$ quantizer.
\end{abstract}

Keywords: variable-length code, companding technique, Gaussian source.

\section{Introduction}

Quantization deals with the digital or, more specifically, binary representation of signals. All the audio and video encoding algorithms typically include a quantization module. Quantization can be: (i) with memory or memoryless, depending upon whether the encoding rules rely or not on past samples; (ii) uniform or nonuniform based on the step-size or the quantization levels employed; (iii) scalar or vector depending upon each sample is quantized individually, or a block of samples is quantized jointly. An algorithm for design 
of the optimal quantizer for a source with known distribution was developed by Lloyd and Max (1960). However, this algorithm is very time consuming for the large number of quantization levels. One solution which overcomes this problem is defined by the companding quantizer model (Jayant and Noll, 1984; Gersho and Gray, 1992; Kondoz, 2004; Sayood, 2006; Hanzo et al., 2007). Quantizers based on the companding model have simple realization structure and performance close to the optimal ones. The design of such quantizers is also more efficient than the one of the Lloyd-Max's quantizers since it does not require the iterative method. This difference is very notable for some commonly used sources including the Gaussian source, we consider in the paper. The reason of considering the Gaussian source is that the first approximation to the shorttime-averaged probability density function (PDF) of speech amplitudes is provided by Gaussian PDF (Jayant and Noll, 1984; Gersho and Gray, 1992; Kondoz, 2004; Sayood, 2006; Hanzo et al., 2007). In case of a high-quality narrowband speech coding, widely used coders are $64 \mathrm{kbit} / \mathrm{s}$ nonuniform scalar quantizers based on segmental logarithmic companding characteristic. This solution is standardized and is known as G.711 quantizer (ITU-T, 1972). Its main disadvantage is that bit rate decrease brings the significant reduction in quality ( $6 \mathrm{~dB}$ per 1 bit). Nowadays, when new bandwidth limited communication systems are deployment, this can be unfavorable. Because of that, more sophisticated speech compression has become essential component in telecommunications. This has prompted extensive research in the area of speech coding during the last decade (Hiwasaki et al., 2006, 2008a, 2008b; Perić and Nikolić, 2007; Nikolić and Perić, 2008; Perić et al., 2009, 2010; Na, 2008, 2011). Namely, fixed-rate scalar quantizers for Gaussian source have already been the topic of our earlier research (Perić et al., 2008; Perić et $a l ., 2010)$. In these two papers, the switched-adaptive quantization technique is utilized for a high-quality quantization of speech signals. The challenge to meet not only G.712 Recommendation for a high-quality speech coding (ITU-T, 2001), but also to provide the gain in the performance over the G.711 quantizer has driven much of the research in this area and several speech coding algorithms have been developed and eventually adopted in international standards (Hiwasaki et al., 2006, 2008a, 2008b).

However, although a great number of a quantizers have been developed to provide an additional enhancement of the speech signal quality, especially for the VoIP applications, where G.711 quantizers are used, there is still a need to continue the research in this field. In this paper we propose switched VLC optimal companding quantizer, composed of two optimal companding quantizers having a different number of representation levels and a different compressor functions. The aim of presented research is to find a simple manner to realize a quantizer system having high-quality performance but maintaining robustness in a wide variance range of input signal. Additionally, our goal is to obtain a flexible compression algorithm, where the flexibility is related to the more sophisticated choice of bit rate while maintaining the necessary quality.

This paper is organized as follows: Section 2 provides a detailed description of the novel switched VLC optimal companding quantizer. In Section 3, we discuss which codebook sizes of two companding quantizers, composing the proposed quantizer, should be chosen in order to satisfy G.712 Recommendation. Section 4 presents the adaptive vari- 
ant of our quantizer model which is tested on the speech coding. Section 5 concludes the paper about the possibilities of the proposed algorithm application in speech processing.

\section{Design of Switched Variable-Length Code Optimal Companding Quantizer}

The set of real numbers $t_{0}, t_{1}, \ldots, t_{N}$, called decision thresholds satisfying

$$
-\infty=t_{0}<t_{1}<\cdots<t_{N-1}<t_{N}=+\infty
$$

and the set $y_{1}, y_{2}, \ldots, y_{N}$, called representation levels satisfying

$$
y_{j} \in \alpha_{j}=\left(t_{j-1}, t_{j}\right], \quad j=1, \ldots, N,
$$

characterize the $N$-level fixed rate scalar quantizer (Jayant and Noll, 1984; Gersho and Gray, 1992; Kondoz, 2004; Sayood, 2006; Hanzo et al., 2007). Every $N$-level fixed rate scalar quantizer is associated with the partition of the set of real numbers $R$ into $N$ cells $\alpha_{j}$. There are several models of scalar quantizers that are based on different quantization techniques. The stucture of nonuniform quantizer, consisting of a compressor, a uniform quantizer and an expandor in cascade, is called compandor (Jayant and Noll, 1984; Gersho and Gray, 1992; Kondoz, 2004; Sayood, 2006; Hanzo et al., 2007). Nonuniform quantization can be achieved by compandor in the following way: compressing the input signal $x$ using a nonuniform compressor function $c(x)$, then by quantizing the compressed signal $c(x)$ employing a uniform quantizer $Q_{u}(c(x))$, and finally by expanding the quantized version of the compressed signal using a nonuniform inverse compressor function $c^{-1}\left(Q_{u}(c(x))\right)$. In the case of the optimal compressor function $c: R \rightarrow[-1,1]$ defined by Judell and Scharf (1986):

$$
c(x)=\left\{\begin{array}{cl}
-1+2 \frac{\int_{-t}^{x} p^{1 / 3}(x) d x}{\int_{-t}^{0} p^{1 / 3}(x) d x+\int_{0}^{t} p^{1 / 3}(x) d x}, & -t<x<0, \\
\int_{0}^{0} p^{1 / 3}(x) d x+\int_{0}^{x} p^{1 / 3}(x) d x & \\
-1+2 \frac{-t}{0} \int_{-t}^{t} p^{1 / 3}(x) d x+\int_{0}^{t} p^{1 / 3}(x) d x &
\end{array}\right.
$$

the decision thresholds $t_{i}$ and the representation levels $y_{i}$ of the companding quantizer are determined by the solutions of the following equations:

$$
c\left(t_{i}\right)=t_{u, i}, \quad i=0,1, \ldots, N, \quad c\left(y_{i}\right)=y_{u, i}, \quad i=1, \ldots, N,
$$

where $t_{u, i}$ are decision thresholds, and $y_{u, i}$ are representation levels of the corresponding uniform quantizer, and $t$ denotes the support region threshold between granular and overload region of an optimal companding quantizer. 
In this paper we consider the Gaussian PDF of the input source, which is one of the most commonly used distributions (Jayant and Noll, 1984; Gersho and Gray, 1992; Kondoz, 2004; Hanzo et al., 2007). The probability density function for a random variable with a Gaussian distribution, mean $\mu$ and variance $\sigma^{2}$ is given by:

$$
p(x)=\frac{1}{\sqrt{2 \pi \sigma^{2}}} e^{-\frac{(x-\mu)^{2}}{2 \sigma^{2}}} .
$$

In the rest of the paper, without loss of generality, we assume that information source is Gaussian source with memoryless property and zero mean value. The PDF of this source is given by:

$$
p(x)=\frac{1}{\sqrt{2 \pi \sigma^{2}}} e^{-\frac{x^{2}}{2 \sigma^{2}}}
$$

Assuming further the unit variance, $\sigma^{2}=1$, by direct evaluation we obtain:

$$
\int_{0}^{t} p^{1 / 3}(x) d x=\sqrt{3}\left(\frac{\pi}{4}\right)^{1 / 3} \operatorname{erf}\left(\frac{t}{\sqrt{6}}\right)
$$

where erf $(x)$ is the error function:

$$
\operatorname{erf}(x)=\frac{2}{\sqrt{\pi}} \int_{0}^{x} e^{-u^{2}} d u
$$

In the rest of the paper we assume symmetry about zero in the quantizer characteristic. This symmetry is an intuitionally expected result when the input has a PDF that is symmetrical about zero. The Gaussian PDF, we consider here, is indeed symmetrical about zero.

Switched quantizer we propose in this paper is composed of two companding quantizers, the inner and the outer one. These two quantizers are designed according to a different optimal compressor functions. Particularly, the inner companding quantizer $Q_{1}$, having $N_{1}$ representation levels, is defined on the inner interval $I=\left[-t_{1}, t_{1}\right]$ while the outer companding quantizer $Q_{2}$, having $N_{2}$ representation levels, is defined on the outer interval $O=\left(-\infty,-t_{1}\right) \cup\left(t_{1},+\infty\right)$. Value $t_{1}$ is called threshold value. For the assumed Gaussian PDF of unit variance the optimal compressor function $c_{1}: I \rightarrow[-1,1]$ of the inner companding quantizer is:

$$
c_{1}(x)=\frac{\operatorname{erf}\left(\frac{x}{\sqrt{6}}\right)}{\operatorname{erf}\left(\frac{t_{1}}{\sqrt{6}}\right)} \operatorname{sgn}(x), \quad|x|<t_{1} .
$$


Further, for the inner companding quantizer the decision thresholds $t_{I, i}$ and the representation levels $y_{I, i}$ are defined by:

$$
\begin{aligned}
& t_{I, i,-}=-t_{I, i,+}=c_{1}^{-1}\left(\frac{2 i-N_{1}}{N_{1}}\right), \quad i=0,1, \ldots, N_{1} / 2, \\
& y_{I, i,-}=-y_{I, i,+}=c_{1}^{-1}\left(\frac{2 i-1-N_{1}}{N_{1}}\right), \quad i=1, \ldots, N_{1} / 2 .
\end{aligned}
$$

Using (9), by direct computation we obtain the following expressions:

$$
\begin{aligned}
& t_{I, i,-}=\sqrt{6} \operatorname{erf}^{-1}\left(\frac{2 i-N_{1}}{N_{1}} \operatorname{erf}\left(\frac{t_{1}}{\sqrt{6}}\right)\right), \quad i=0,1, \ldots, N_{1} / 2, \\
& y_{I, i,-}=\sqrt{6} \operatorname{erf}^{-1}\left(\frac{2 i-1-N_{1}}{N_{1}} \operatorname{erf}\left(\frac{t_{1}}{\sqrt{6}}\right)\right), \quad i=1, \ldots, N_{1} / 2 .
\end{aligned}
$$

The decision thresholds of the outer companding quantizer $Q_{2}$ in each of the two symmetric intervals composing the outer region $O$ are ordered such that we have $-\infty=$ $t_{O, 0,-}<t_{O, 1,-}<\ldots<t_{O, N_{2} / 2,-}=-t_{1}$ and $t_{1}=t_{O, N 2 / 2,+}<t_{O, N 2 / 2-1,+}<$ $\ldots<t_{O, 0,+}=+\infty$. Similarly, one can define the order of the corresponding positive and negative representation levels $y_{O, i,+}$ and $y_{O, i,-}, i=1, \ldots, N_{2} / 2$. The optimal compressor function $c_{2}(x): O \longrightarrow[-1,1]$ defining the outer companding quantizer $Q_{2}$ can be calculated from (3), where $0 \rightarrow t_{1}$ and $t=+\infty$ :

$$
c_{2}(x)= \begin{cases}-\frac{\operatorname{erf}(x / \sqrt{6})-\operatorname{erf}\left(t_{1} / \sqrt{6}\right)}{1-\operatorname{erf}\left(t_{1} / \sqrt{6}\right.}, & -\infty<x<-t_{1}, \\ \frac{\operatorname{erf}(x / \sqrt{6})-\operatorname{erf}\left(t_{1} / \sqrt{6}\right)}{1-\operatorname{erf}\left(t_{1} / \sqrt{6}\right)}, & t_{1}<x<\infty .\end{cases}
$$

The decision thresholds and the representation levels of the companding outer quantizer $Q_{2}$ are computed from the following relations:

$$
\begin{aligned}
& t_{O, i,-}=-t_{O, i,+}=c_{2}^{-1}\left(\frac{2 i-N_{2}}{N_{2}}\right), \quad i=0,1, \ldots, N_{2} / 2 \\
& y_{O, i,-}=-y_{O, i,+}=c_{2}^{-1}\left(\frac{2 i-1-N_{2}}{N_{2}}\right), \quad i=1, \ldots, N_{2} / 2 .
\end{aligned}
$$

By an explicit computation using the relation (14) we obtain the following expressions:

$$
\begin{aligned}
& t_{O, i,-}=\sqrt{6} \operatorname{erf}^{-1}\left(\frac{2 i-N_{2}}{N_{2}}\left(1-\operatorname{erf} \frac{t_{1}}{\sqrt{6}}\right)-\operatorname{erf} \frac{t_{1}}{\sqrt{6}}\right), \\
& i=0,1, \ldots N_{2} / 2 \\
& y_{O, i,-}=\sqrt{6} \operatorname{erf}^{-1}\left(\frac{2 i-1-N_{2}}{N_{2}}\left(1-\operatorname{erf} \frac{t_{1}}{\sqrt{6}}\right)-\operatorname{erf} \frac{t_{1}}{\sqrt{6}}\right), \\
& i=1, \ldots N_{2} / 2 .
\end{aligned}
$$


Finally we can write decision thresholds and representation levels of the joint switched optimal companding quantizer $Q$ as follows:

$$
\begin{aligned}
t= & \left(-\infty, \ldots t_{O, N 2 / 2-1,-},-t_{1}, t_{I, 1}, \ldots, t_{I, N 1 / 2-1}, t_{1}, t_{O, N 2 / 2-1,+}\right. \\
& \ldots+\infty) \\
y= & \left(y_{O, 0,-}, \ldots y_{O, N 2 / 2-1,-}, y_{I, 0}, y_{I, 1}, \ldots, y_{I, N 1 / 2-1}, y_{O, N 2 / 2-1,+}\right. \\
& \left.\quad \ldots y_{O, 0,+}\right)
\end{aligned}
$$

For the proposed quantizer, the first bit of the codeword corresponding to the source sample $x$ is equal 1 if the sample belongs to the inner region ( $\left.x \in I=\left[-t_{1}, t_{1}\right]\right)$, otherwise it is equal 0 . Other $\log _{2} N_{1}$ (or $\log _{2} N_{2}$ ) bits of the codeword are formed by an index of the quantization cell $x$ belongs to. Since the length of the codeword is $k_{1}+1$ or $k_{2}+1,\left(N_{i}=2^{k_{i}}\right)$, depending on whether $x \in I$ or not, our quantizer is a variable-length quantizer (VLC - variable-length coder). In general, with variable length quantizers compression is achieved by assigning shorter codewords to the more frequent symbols and longer codewords to the less frequent ones (Gersho and Gray, 1992; Hanzo et al., 2007). The average bit rate of the considered quantizer can be computed by:

$$
R(Q)=P_{1}\left(\log _{2} N_{1}+1\right)+P_{2}\left(\log _{2} N_{2}+1\right)
$$

where $P_{i}, i=1,2$, are probabilities that one signal sample belongs to the inner and to the outer region, respectively. The additional bits, in previous formula, carries necessary side information for decoding, i.e., which region, and which number of quantization levels is used in encoding process. Since:

$$
\begin{aligned}
& P_{1}=\int_{-t_{1}}^{t_{1}} p(x) d x \\
& P_{2}=1-P_{1}
\end{aligned}
$$

for the average bit rate we obtain:

$$
R(Q)=\operatorname{erf}\left(\frac{t_{1}}{\sqrt{2}}\right)\left(\log _{2} N_{1}+1\right)+\left(1-\operatorname{erf}\left(\frac{t_{1}}{\sqrt{2}}\right)\right)\left(\log _{2} N_{2}+1\right)
$$

Further, we can express threshold $t_{1}$ as a function of $R, N_{1}$ and $N_{2}$, in the following way:

$$
t_{1}=\sqrt{2} \operatorname{erf}^{-1}\left(\frac{R-1-\log _{2} N_{2}}{\log _{2} N_{1}-\log _{2} N_{2}}\right)
$$

It is obvious that the choice of the threshold $t_{1}$ has an effect on the probabilities $P_{1}$ and $P_{2}$, and thus on $R(Q)$ which is therefore much more sophisticated $R$ and can be easily changed in relation to the case where $R$ is obtained as $R=\log _{2}\left(N_{1}+N_{2}\right)$. For instance, 
one can determine the threshold $t_{1}$ that provides a high-quality quantization specified by the G.712 Recommendation (ITU-T, 2001).

The quality of the $N$-level scalar quantizer $Q$, for a source that is characterized as a continuous random variable with PDF $p(x)$, is commonly measured by the distortion defined by the expected mean square error between original and quantized signal (Jayant and Noll, 1984; Gersho and Gray, 1992; Kondoz, 2004; Sayood, 2006; Hanzo et al., 2007):

$$
D(Q)=E(X-Q(X))^{2}=\sum_{i=1}^{N} \int_{t_{i-1}}^{t_{i}}\left(x-y_{i}\right)^{2} p(x) d x .
$$

The $N$-level quantizer $Q^{*}$ is optimal for the source $X$, if there is no other $N$-level quantizer $Q$, such that $D(Q)<D\left(Q^{*}\right)$. Denote by $D_{1}$ and $D_{2}$ the distortions of the inner and the outer quantizer. Also denote by $N_{1}$ and $N_{2}$ the number of representation levels of the inner and the outer quantizer, respectively. In this model, distortions $D_{1}$ and $D_{2}$ can be approximated using Bennett's integral as follows (Bennett, 1948):

$$
\begin{aligned}
& D_{1}=D\left(Q_{1}\right)=\frac{2}{3 N_{1}{ }^{2}}\left(\int_{0}^{t_{1}} p^{1 / 3}(x) d x\right)^{3}, \\
& D_{2}=D\left(Q_{2}\right)=\frac{2}{3 N_{2}{ }^{2}}\left(\int_{t_{1}}^{\infty} p^{1 / 3}(x) d x\right)^{3} .
\end{aligned}
$$

Finally, total distortion $D=D_{1}+D_{2}$ for the assumed Gaussian source of unit variance can be represented, using (6), (27) and (28), as the function of $t_{1}, N_{1}$ and $N_{2}$ as follows:

$$
D(Q)=\frac{\sqrt{3} \pi}{2{N_{1}}^{2}} \operatorname{erf}\left(t_{1} / \sqrt{6}\right)^{3}+\frac{\sqrt{3} \pi}{2{N_{2}}^{2}}\left(1-\operatorname{erf}\left(t_{1} / \sqrt{6}\right)\right)^{3} .
$$

It is easy to prove that there exists one global minimum, which is the solution of the equation $D^{\prime}\left(t_{1}\right)=0$, and we apply the Newton iterative method to find the numerical solution.

\section{Numerical Results}

Quality of a quantized signal is often specified in terms of Signal to Quantization Noise Ratio $(S Q N R)$, which is directly obtained from the distortion $D$ using the following relation (Jayant and Noll, 1984; Gersho and Gray, 1992; Kondoz, 2004; Sayood, 2006; Hanzo et al., 2007):

$$
S Q N R=10 \log \left(\sigma^{2} / D\right) .
$$


Table 1

Values of $t_{1}, R$, and $S Q N R$ for the presented model and a different value of $N_{1}$ and $N_{2}$

\begin{tabular}{rrlll}
\hline$N_{1}$ & $N_{2}$ & $t_{1}$ & $R$ [bit/sample] & $S Q N R[\mathrm{~dB}]$ \\
\hline 32 & 128 & 0.438810 & 7.32160 & 39.7356 \\
16 & 128 & 0.241985 & 7.42637 & 38.8204 \\
32 & 64 & 0.746042 & 6.45564 & 35.2986 \\
16 & 64 & 0.438810 & 6.32160 & 33.7150 \\
\hline
\end{tabular}

Table 2

Values of $t_{1}^{34}$ and $R^{34}$ for the presented model such that $S Q N R=34 \mathrm{~dB}$

\begin{tabular}{rrll}
\hline$N_{1}$ & $N_{2}$ & $t_{1}^{34}$ & $R^{34}$ [bit/sample] \\
\hline 32 & 128 & 1.231717 & 6.43611 \\
16 & 128 & 0.712567 & 6.42834 \\
32 & 64 & 1.136107 & 6.25591 \\
16 & 64 & 0.438810 & 6.32160 \\
\hline
\end{tabular}

For $R \in\left(\min \left(\log _{2} N_{1}, \log _{2} N_{2}\right)+1, \max \left(\log _{2} N_{1}, \log _{2} N_{2}\right)+1\right)$, using equations (25), (29) and (30), we can get the $S Q N R$ value as a function of $R$ for our model. Computed values of the threshold $t_{1}$ and $S Q N R$ for the presented model, designed for the Gaussian source of unit variance and a different values of $N_{1}$ and $N_{2}$, are given in the Table 1. As it can be noticed from the Table 1 a smaller size of the inner companding quantizer codebook is assumed, i.e., $N_{1}<N_{2}$. This assumption is introduced in order to assign shorter codewords to the high probability low amplitude samples belonging to the inner interval. In such a manner the basic principe of VLC quantization is fulfilled. What also can be noted from the first and the second raw in the Table 1 is that the increase of the bit rate do not result in $S Q N R$ increase. The explanation for this behavior can be found in the expression for the bit rate (24), where in addition to the influence of codebook sizes and side information we have a threshold $t_{1}$ effect manifested through the probability of coding with corresponding companding quantizer. Recall that the listed values of threshold $t_{1}$ are calculated by applying the Newton iterative method to solve the equation $D^{\prime}\left(t_{1}\right)=0$. Define $t_{1}^{34}$ as a value of threshold such that $S Q N R$ of the proposed quantizer, designed for the Gaussian source of unit variance, is $34 \mathrm{~dB}$. Denote by $R^{34}$ corresponding average bit rate. Table 2 presents values of $t_{1}^{34}$ and $R^{34}$ we have calculated for a different values of $N_{1}$ and $N_{2}$. From the Table 2 one can perceive that our quantizer designed for the Gaussian source of unit variance, can provide a high-quality quantization with about $6.25 \mathrm{bit} / \mathrm{sample}$. Moreover, it can be noticed that in comparison to the classical fixed-rate companding quantizer, our quantizer enables more sophisticated bit rate reduction. Figure 1 shows $S Q N R$ of our quantizer as a function of the signal variance in the case of a different choice of the threshold $t_{1}^{34}$. It is interesting to observe from Fig. 1 that for a different threshold $t_{1}^{34}$ the maximum $S Q N R$ is achieved at a different variances $\sigma_{i}^{2}$. Particularly, as the threshold value is higher, the maximum of $S Q N R$ characteristic 


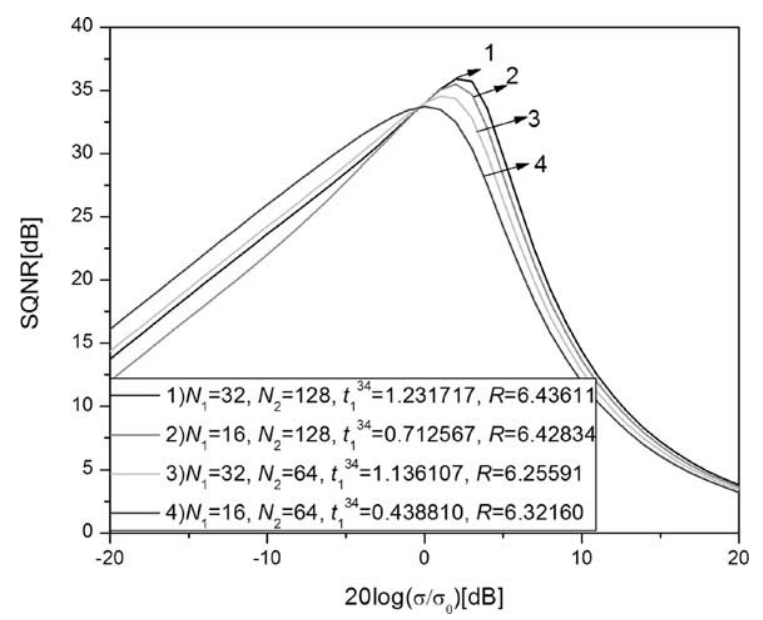

Fig. 1. $S Q N R$ as a function of the signal variance for a different values of $N_{1}, N_{2}$ and $t_{1}^{34}$.

is moved toward right, i.e., toward higher values of variances. $S Q N R$ characteristic is distinctly asymmetrical and rapidly decreases from its maximum, such that $S Q N R$ varies a lot in the observed variance range. Generally, any of non-adaptive (fixed) quantizers designed for the particular variance is not suitable for quantization of non-stationary signals that, as well as speech signals, have changing variance characteristic (Jayant and Noll, 1984; Gersho and Gray, 1992). In such situations, as it can be noticed from Fig. 1 and as it has been observed in $\mathrm{Na}(2008,2011)$ the variance mismatch between the input signal's variance and the variance for which the quantizer is designed may occur. However, in short intervals the properties of the speech signal remain roughly constant, hence, it can be viewed as a local-stationary signal (Jayant and Noll, 1984; Gersho and Gray, 1992; Hanzo et al., 2007). Accordingly, in order to process speech efficiently it is necessary to use same kind of adaptation or system that works on frame by frame basis. For that reason, in the next section, we consider forward adaptive version of the proposed quantizer model and its application in speech coding.

\section{Application in Speech Coding}

We have tested our coding scheme on the speech coding. The sample signal is taken from the base which is derived from the TIMIT corpus (Garofolo et al., 1993). The TIMIT corpus of speech has been designed to provide speech data for the acquisition of acoustic-phonetic knowledge and for the development and evaluation of automatic speech recognition systems. For the purpose of testing, we consider the adaptive version of our model. We divide the input signal into the frames and for each frame we estimate the signal variance and normalize all samples before coding. Consider the $n$ samples of the input signal $x_{1}, x_{2}, \ldots, x_{n}$ and assume that signal samples are divided in $F$ frames each consisted of $M$ samples. Furthermore denote by $x_{i, j}$ the $j$ th sample of the $i$ th frame 
( $i=0, \ldots, F-1$ and $j=0, \ldots, M-1)$, i.e., $x_{i, j}=x_{i M+j}$. In the $i$ th frame signal variance is estimated using $\sigma_{i}^{2}=\frac{1}{M} \sum_{j=0}^{M-1} x_{i, j}^{2}$. The source samples are then normalized and sent to the quantizer. When received, the signal has to be denormalized by the same value of the squared root of the quantized variance that is used for normalization. For that purpose, we also need to transmit the information about the signal variance $\sigma_{i}^{2}$. It is quantized using $N_{g}$ level log-uniform scalar quantizer. We consider the log-uniform scalar quantizer for variance quantization rather than the uniform scalar quantizer, since we have demonstrated that it could provide higher value of $S Q N R$ (Nikolić and Perić, 2008). Signal samples are normalized to $\bar{x}_{i, j}=x_{i, j} / \tilde{\sigma}_{i}$, where $\tilde{\sigma}_{i}$ is squared root of the quantized signal variance, and then sent to the quantizer.

The representation levels and the decision thresholds of log-uniform quantizer $Q_{l u}(\sigma)$ are defined as:

$$
\begin{aligned}
& \log \left(y_{l u, i}\right)=\log \left(\sigma_{\min }\right)+\frac{2 i-1}{2 N_{g}} \log \left(\frac{\sigma_{\max }}{\sigma_{\min }}\right), \quad i=1,2, \ldots, N_{g}, \\
& \log \left(t_{l u, i}\right)=\log \left(\sigma_{\min }\right)+\frac{i}{N_{g}} \log \left(\frac{\sigma_{\max }}{\sigma_{\min }}\right), \quad i=0,1,2, \ldots, N_{g}
\end{aligned}
$$

where $\sigma_{\min }^{2}$ and $\sigma_{\max }^{2}$ are respectively maximum and minimum possible values of the signal variance. In other words, log-uniform quantizer is the uniform quantizer in decibels scale. We consider the dynamic range of the variance $\left(20 \log \left(\sigma_{\max } / \sigma_{\min }\right)[\mathrm{dB}]\right)$ of $40 \mathrm{~dB}$, $N_{g}=16$ and $N_{g}=32$ levels of log-uniform quantizer. The bit rate $R$ corresponding to the proposed adaptive version is $R=R_{f i x}+\log _{2} N_{g} / M$, where $R_{f i x}$ refers to the number of bits per sample required for the fixed quantizer (24), while $\log _{2} N_{g} / M$ define the number of bits per frame having length $M$ that is required for transmission of the side information for adaptive version. Figure 2 shows theoretical dependencies of the $S Q N R$

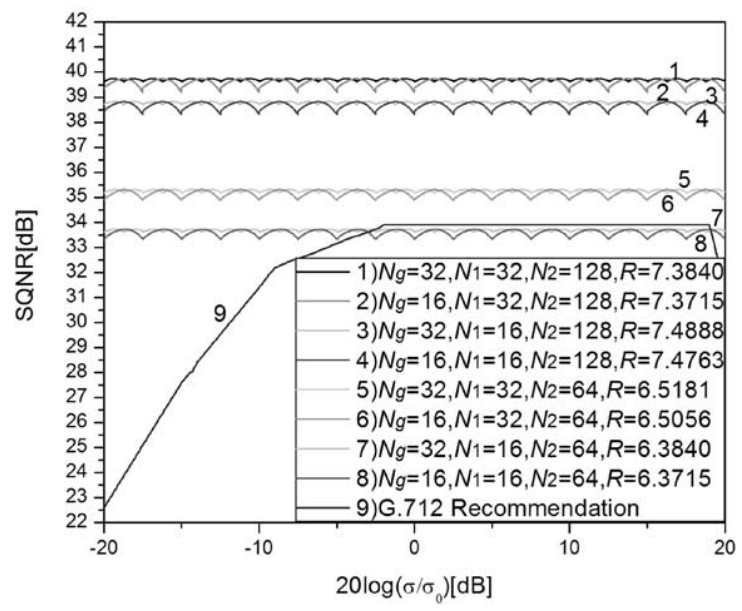

Fig. 2. Theoretical dependence of the $S Q N R$ as a function of signal variance $\sigma^{2}$ (in decibels) for the presented adaptive model $\left(N_{g}=16,32\right)$. 
Table 3

\begin{tabular}{|c|c|c|c|c|}
\hline$N_{1}$ & $N_{2}$ & $N_{g}$ & $R$ [bit/sample] & $S Q N R_{\text {avg }}[\mathrm{dB}]$ \\
\hline 32 & 128 & 32 & 7.3840 & 39.6952 \\
\hline 32 & 128 & 16 & 7.3715 & 39.5526 \\
\hline 16 & 128 & 32 & 7.4888 & 38.7815 \\
\hline 16 & 128 & 16 & 7.4763 & 38.6418 \\
\hline 32 & 64 & 32 & 6.5181 & 35.2669 \\
\hline 32 & 64 & 16 & 6.5056 & 35.1393 \\
\hline 16 & 64 & 32 & 6.3840 & 33.6873 \\
\hline 16 & 64 & 16 & 6.3715 & 33.5656 \\
\hline
\end{tabular}

Table 4

Values of $t_{1}^{34}$, and $R^{34}$, for the adaptive version of the presented model such that $S Q N R_{\min }>34 \mathrm{~dB}$, i.e., satisfying G.712 Recommendation

\begin{tabular}{lrlll}
\hline$N_{1}$ & $N_{2}$ & $N_{g}$ & $t_{1}^{34}$ & $R^{34}$ [bit/sample] \\
\hline 32 & 128 & 32 & 1.22506 & 6.50361 \\
32 & 128 & 16 & 1.25132 & 6.47164 \\
16 & 128 & 32 & 0.72497 & 6.46791 \\
16 & 128 & 16 & 0.73560 & 6.43592 \\
32 & 64 & 32 & 1.14438 & 6.31497 \\
32 & 64 & 16 & 1.13841 & 6.30495 \\
\hline
\end{tabular}

values as a function of signal variance $\sigma^{2}$ (in decibels) for presented adaptive quantizer model ( $\left.M=80, N_{g}=16,32\right)$. From the Fig. 2 one can notice that our multi-resolution quantizer provides a different level of signal quality, i.e., of the $S Q N R$. Moreover, one can perceive that by increasing the level number of log-uniform quantizer, the bit rate is slightly increased, but the $S Q N R$ characteristic is significantly flattened. Particularly, one can observe that in the considered variance range $S Q N R$ is almost constant for $N_{g}=32$. Accordingly, $N_{g}=32$ is a good choice for the number of log-uniform variance quantizer levels. Using the basic definition for the average $S Q N R$ :

$$
S Q N R_{a v g}=\frac{1}{K} \sum_{i=1}^{K} 10 \log \left(\sigma_{i}^{2} / D\left(\sigma_{i}\right)\right)
$$

where $K$ defines the number of the particular variances that are calculated in the assumed variances range of $40 \mathrm{~dB}$, we have calculated values presented in the Table 3 for adaptive version of our quantizer. It has already been mentioned and explained how the codebook sizes, amount of the side information and the threshold choice influence on $S Q N R$ and $R$ of the corresponding non-adaptive quantizer. The same conclusion can be derived for the adaptive version of our quantizer model. In Table 4 are given values of $t_{1}^{34}$ and $R^{34}$ 


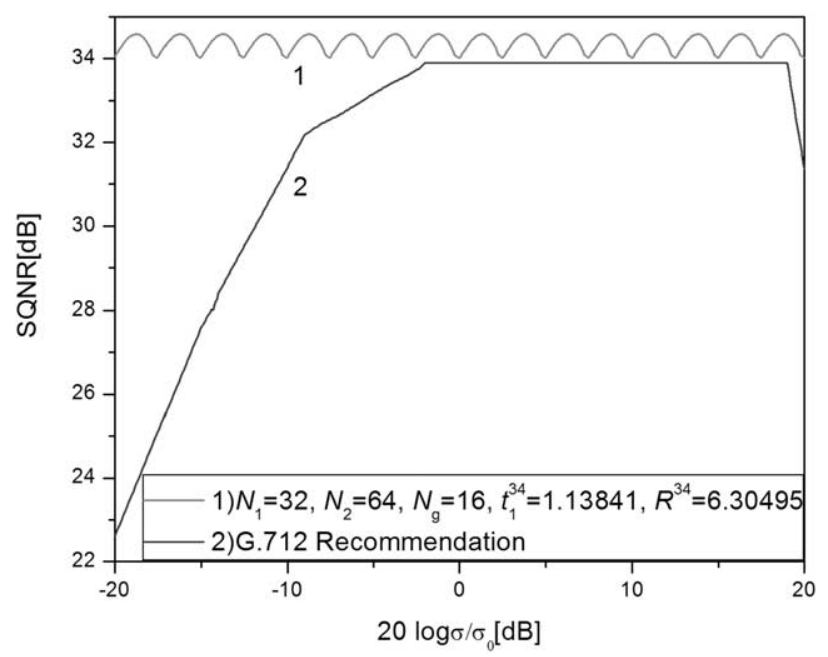

Fig. 3. Adaptive version of the presented model with values of $t_{1}^{34}$ and $R^{34}$ such that $S Q N R_{\min }>34 \mathrm{~dB}$, i.e., satisfying G.712 Recommendation.

[bit/sample] for which the minimum value of $S Q N R$ for adaptive version of our model does not fall below $34 \mathrm{~dB}$. These numerical results obtained to maintain a high-quality quantization (ITU-T, 2001) the best reflect gains in compression that ranges up to 1.7 bit/sample over the G.711 quantizer. Fig. 3 shows $S Q N R$ characteristic of the adaptive version of presented model designed for $t_{1}^{34}=1.13841$ and the bit rate of $R^{34}=6.30495$ such that $S Q N R_{\min }>34 \mathrm{~dB}$, i.e., such that satisfies G.712 Recommendation. This adaptive version of our quantizer model in the considered wide variance range provides a highquality quantization along with the compression of about 1.7 bit/sample over the G.711 quantizer (ITU-T, 1972). Moreover, our quantizer model achieves the compression of $6.7-6.3=0.4$ bit/sample over the quantizer proposed in Perić et al. (2010). Particularly, in Perić et al. (2010) additional gain in the compression is achieved applying the lossless compression technique where the resulting gain over the G.711 quantizer amount to $8-6.37=1.63 \mathrm{bit} / \mathrm{sample}$. This compression gain is smaller than the one we achieved in this paper without applying some of the lossless compression techniques. Fig. 4 shows the experimental $S Q N R$ values as a function of $\sigma^{2}$ for adaptive variant of our model, and different values of $N_{1}, N_{2}$, and $N_{g}$. For the purpose of the experiment, we assumed the frame size of $M=80$ and total $F=800$ frames. We determined the experimental values $S Q N R e x_{i}$ for each frame $i=0,1, \ldots, F-1$. In Fig. 4 we show the input signal (the first upper graph) and $S Q N R e x_{i}$ values (the second lower graph). Note that the $x$ scale, on the lower graphs, still represents the index of the sample (not the frame). The average $S Q N R$ value of all frames is equal to $S Q N R e x=\frac{1}{F} \sum_{i=0}^{F-1} S Q N R e x_{i}$. Comparing the theoretical and experimental values of the average $S Q N R$ for the several different values of the average bit rate $R$, one can calculated that there is a good agreement between the theory and experiment in all of the considered cases. 

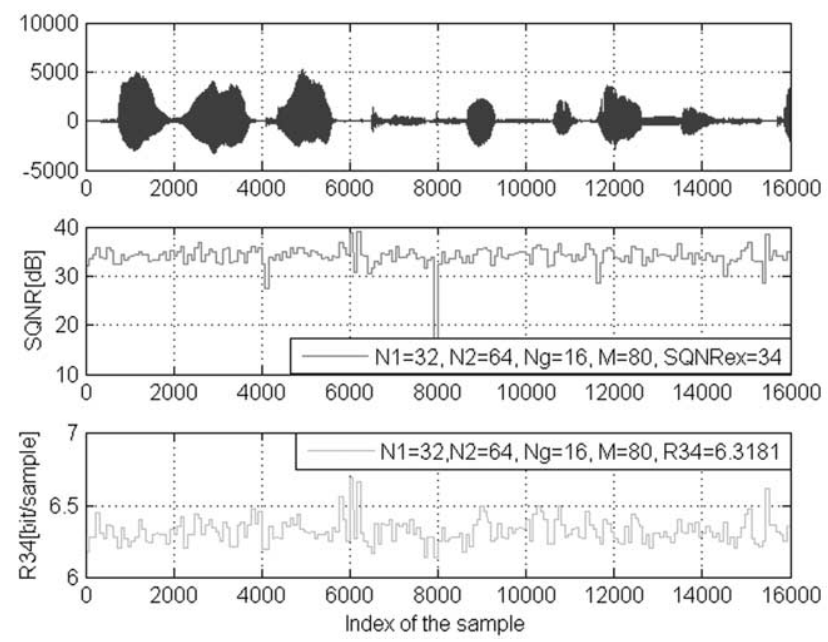

Fig. 4. An input speech signal (the first graph), experimental values of $S Q N R e x_{i}$ (the second graph) and $R_{i}^{34}$ (the third graph) for the adaptive version of the presented model.

\section{Conclusions}

In this paper we have presented and analyzed performances of the new model of switched VLC optimal companding quantizer. The model is designed for a high-quality scalar quantization of Gaussian source in a wide variance range of input signal. According the analysis of the codebook size influence and the influence of the threshold $t_{1}$ choice on SQNR it has been concluded that adaptive version of our quantizer model satisfies G.712 Recommendation, providing the compression of about $1.7 \mathrm{bit} / \mathrm{sample}$ over the G.711 quantizer (ITU-T, 1972), so it can be applied for a high-quality coding of speech signals. It has also been demonstrated that, in comparison to the fixed-rate scalar companding quantizers, the proposed multi-resolution quantizer provides much more sophisticated choice of the bit rate depending on the desired level of speech signal quality. Finally, good agreement between the theoretical and experimental values of the average $S Q N R$ has been ascertained. Our quantizer gives a very simple realization structure and highquality performances, hance it can be very useful in particular high-quality quantization of signals having Gaussian PDF.

\section{References}

Bennett, W.R. (1948). Spectra of quantized signals. The Bell Systems Technical Journal, 27, 446-472.

Garofolo, J.S., Lamel, F.L., Fisher, W.M., Fiscus, J.G., Pallett, D.S., Dahlgren, N.L. (1993). The DARPA TIMIT acoustic-phonetic continuous speech corpus. CD-ROM: NTIS.

Gersho, A., Gray, R.M. (1992). Vector Quantization and Signal Compression. Kluwer, Norwell.

Hanzo, L., Somerville, C., Woodard, J. (2007). Voice and Audio Compression for Wireless Communications. Wiley/IEEE Press, New York.

Hiwasaki, Y., Ohmuro, H., Mori, T., Kurihara, S., Kataoka, A. (2006). A G.711 embedded wideband speech coding for VoIP conferences. Transactions on Info and Systems (IEICE), E89-D(9), 2542-2552. 
Hiwasaki, Y. et al. (2008). G.711: a wideband extension to ITU-T G.711. In: Proceedings of EUSIPCO'08, Lausanne, Switzerland.

Hiwasaki, Y., Mori, T., Sasaki, S., Ohmuro, H., Kataoka, A. (2008). A wideband speech and audio coding candidate for ITU-T G.711 WBE standardization. In: ICASSP 2008.

ITU-T, Recommendation G.711. (1972). Pulse Code Modulation (PCM) of Voice Frequencies.

ITU-T, Recommendation G.712. (2001). Transmission Performance Characteristics of Pulse Code Modulation Channels.

Jayant, N.S., Noll, P. (1984). Digital Coding of Waveforms. Prentice-Hall, New Jersey.

Judell, N., Scharf, L. (1986). A simple derivation of Lloyd's classical result for the optimum scalar quantizer. IEEE Transactions on Information Theory, 32(2), 326-328.

Kondoz, A. (2004). Digital Speech Coding for Low Bit Rate Communication Systems. Wiley, New York.

Max, J., (1960). Quantizing for minimum distortion. In: IRE, Transactions on Information Theory, Vol. IT-6, pp. $7-12$

Na, S. (2008). Asymptotic formulas for mismatched fixed-rate minimum MSE Laplacian quantizers. Signal Processing Letters (IEEE), 15, 13-16.

$\mathrm{Na}$, S. (2011). Asymptotic formulas for variance-mismatched fixed-rate scalar quantization of a Gaussian source. IEEE Transactions on Signal Processing, 59(5), 2437-2441.

Nikolić, J., Perić, Z. (2008). Lloyd-Max's algorithm implementation in speech coding based on forward adaptive technique. Informatica, 19(2), 255-270.

Perić, Z., Nikolić, J. (2007). An effective method for initialization of Lloyd-Max's algorithm of optimal scalar quantization for Laplacian source. Informatica, 18(2), 279-288.

Perić, Z., Petković, M., Dincić, M. (2009). Simple compression algorithm for memoryless Laplacian source based on the optimal companding technique. Informatica, 20(1), 99-114.

Perić, Z., Savić, M., Dincić, M., Denić, D., Prascević, M. (2010). Forward adaptation of novel semilogarithmic quantizer and lossless coder for speech signals compression. Informatica, 21(3), 375-391.

Perić, Z., Mosić, A., Panić, S. (2008). Robust and switched nonuniform scalar quantization of Gaussian source in a wide dinamic range of power. Automatic Control and Computer Science, 42(6), 334-341.

Perić, Z., Nikolić, J., Mosić, A., Panić, S. (2010). A switched-adaptive quantization technique using $\mu$-law quantizers. Information Technology and Control, 39(4), 317-320.

Sayood, K. (2006). Introduction to Data Compression. Elsevier, Amsterdam. 
Z. H. Perić was born in Nis, Serbia, in 1964. He received the BS degree from the Faculty of Electronic Engineering, Nis, Serbia, in 1989, and MS degree from the University of Nis, in 1994. He received the PhD degree from the University of Nis, in 1999. He is currently a full professor at the Department of Telecommunications and vicedean of the Faculty of Electronic Engineering, University of Nis, Serbia. His current research interests include the information theory, source and channel coding and signal processing. He is particularly working on scalar and vector quantization techniques in speech and image coding. He is author and coauthor in over 150 papers in digital communications. Dr. Perić has been a reviewer for IEEE Transactions on Information Theory and The International Journal for Computation and Mathematics in Electrical Engineering (COMPEL). He is member of the editorial board of the journal Electronics and Electrical Engineering .

J.R. Nikolić was born in Prokuplje, Serbia, in 1978. She received the BS and MS degrees from the Faculty of Electronic Engineering, Nis, Serbia, in 2003 and 2006, respectively. She received the $\mathrm{PhD}$ degree from the University of Nis in 2011. She is currently a teaching assistant at the Faculty of Electronic Engineering at the Department of Telecommunications. Her current research interests include the information theory, source and channel coding and signal processing. She has published over 50 papers on the above subjects.

A.V. Mosić was born in Leskovac, Serbia, in 1983. He received MS degree in electrical engineering from the Faculty of Electronic Engineering, Nis, Serbia, in 2007. He joined the Department of Telecommunications, Faculty of Electronic Engineering, Nis in 2008 as research assistant on joint project between Faculty of Electronic Engineering and Ministry of Science Republic of Serbia. His current research interests include the information theory, source and channel coding and signal processing. He has published over 20 papers (about 10 of them in peer-reviewed international journals) on the above subjects.

M.D. Petković was born in Niš, Serbia, in 1984. He graduated in mathematics and computer science at the Faculty of Science and Mathematics, Nišs, Serbia in 2006. He also graduated in telecommunications at Faculty of Electronic Engineering, Nis, Serbia in 2007. Also he received his $\mathrm{PhD}$ degree in computer science from the University of Niš in 2008. Currently he is an assistant professor in computer science at the Faculty of Science and Mathematics, University of Niš, Serbia. His research interests include the theory and computation of matrix generalized inverses, computational geometry, source and channel coding, symbolic computation and optimization methods. He is the author of about 50 papers (about 30 of them in peer-reviewed international journals). He is supported by the research project 174013 of the Serbian Ministry of Science and Education. 


\title{
Fiksuotas ir adaptyvus kintamo kodinio žodžio ilgio kvantatorius Gauso šaltiniams be atminties
}

\author{
Zoran H. PERIĆ, Jelena R. NIKOLIĆ, Aleksandar V. MOSIĆ, Marko D. PETKOVIĆ
}

Straipsnyje nagrinejjamas kintamo kodo ilgio skaliarinio kvantavimo uždavinys. Autoriai siūlo kintamo kodo ilgio kvantatoriu sudaryti iš dviejų nuosekliai sujungtu skaliariniu kvantatorių, kiekvienas kuriu yra optimizuotas darbui su Gauso šaltiniais be atminties. Šie kvantatoriai naudoja skirtingas kodines knygas bei skirtingas suspaudimo funkcijas. Straipsnyje autoriai ivertino naudojamu kodiniu knygu dydžio įtaką kvantavimo kokybei ir parodè pasiūlytojo kvantatoriaus pranašumą koduojant kalbos signalus prieš fiksuoto kodo ilgio kvantatorius. 